\title{
New Approximations and Calibration Methods to Provide Routine Real-Time Polarimetry on JET
}

\author{
Michela Gelfusa, Andrea Murari, Pasquale Gaudio, Alexandru Boboc, Didler Mazon, Fabio Avino, \\ Ivan Lupelli, F. P. Orsitto, O. Tudisco, and JET-EFDA Contributors*
}

\begin{abstract}
The increasing importance of providing reliable polarimetric measurements in real time, for both machine protection and plasma control, has motivated the development of a quick version of the calibration algorithms for JET polarimeter. This new code, which interprets the calibration procedure performed before each shot, is based on a physical equivalent model of the diagnostic optical path and is valid for any operational regime of JET. It provides results before the plasma breakdown, and, with its estimates of the optical paths parameters, the polarimetric measurements have an accuracy more than sufficient for real-time purposes. New approximate equations have been validated in order to obtain the line integrated density from the newly calibrated horizontal chords, so that also these polarimetric measurements can also be used for density feedback and machine protection. The availability of reliable polarimetric measurements in real time opens new perspectives also to the determination of the plasma boundary, the magnetic equilibrium, and their use in advanced feedback control schemes.
\end{abstract}

Index Terms - Line integrated density, polarimetry, real-time.

\section{INTRODUCTION}

$\mathbf{T}$ HE IMPORTANCE of providing reliable measurements for feedback control of Tokamak plasmas has become increasingly evident in the last years. The operation of the

Manuscript received June 6, 2011; revised August 20, 2011; accepted January 11, 2012. Date of publication February 23, 2012; date of current version April 11, 2012. This work, supported by the European Communities under the contract of Association between EURATOM and ENEA/CEA, was carried out under the framework of the European Fusion Development Agreement. The views and opinions expressed herein do not necessarily reflect those of the European Commission.

M. Gelfusa, P. Gaudio, and I. Lupelli are with the JET-EFDA, Culham Science Centre, OX14 3DB, Abingdon, U.K. and also with the Associazione EURATOM-ENEA-University of Rome "Tor Vergata," 00133 Roma, Italy (e-mail: gelfusa@ing.uniroma2.it; gaudio@ing.uniroma2.it; ivan.lupelli@ uniroma2.it).

A. Murari is with the JET-EFDA, Culham Science Centre, OX14 3DB, Abingdon, U.K. and also with the Consorzio RFX Associazione EURATOMENEA per la Fusione, 4-35127 Padova, Italy (e-mail: amura@jet.uk).

A. Boboc is with the JET-EFDA, Culham Science Centre, OX14 3DB, Abingdon, U.K. and also with the EURATOM/CCFE Fusion Association, Culham Science Centre, Abingdon, OX14 3DB, U.K. (e-mail: Alexandru.Boboc@ ccfe.ac.uk).

D. Mazon is with the JET-EFDA, Culham Science Centre, OX14 3DB, Abingdon, U.K. and also with the Association EURATOM-CEA, CEA Cadarache, 13108 Saint-Paul-lez-Durance, France (e-mail: didier.mazon@ jet.uk).

F. Avino, F. P. Orsitto, and O. Tudisco are with the JET-EFDA, Culham Science Centre, OX14 3DB, Abingdon, U.K. and also with the Associazione EURATOM-ENEA-CR Frascati, 00044 Frascati, Italy (e-mail: fabio_avino@ libero.it; orsitto@frascati.enea.it; tudisco@ frascati.enea.it).

* See the Appendix of F. Romanelli et al., Proceedings of the 23rd IAEA Fusion Energy Conference 2010, Daejeon, Korea

Color versions of one or more of the figures in this paper are available online at http://ieeexplore.ieee.org.

Digital Object Identifier 10.1109/TPS.2012.2186641 next generation of devices, such as JET with a metallic wall and ITER, will impose even more stringent requirements on the diagnostics for real time. More quantities will have to be provided with higher accuracy and more reliably. The use of these measurements ranges from simple single input single output schemes for machine protection to more involved multiple inputs multiple outputs systems for sophisticated control of advance plasma experiments (see Section VI).

One of the diagnostics, whose data in real time would really open new perspectives to control, is polarimetry. Thermonuclear plasmas are active and anisotropic media, which strongly affect the propagation of electromagnetic radiation, depending on their parameters. The measurement of the change in the polarization state (Faraday rotation and Cotton-Mouton effect) of a laser beam probing plasma can therefore provide very useful information about various physical quantities, crucial for both the understanding of the physics and the control of the configuration. The measurement of the Faraday rotation is one of the very few techniques to probe the magnetic fields inside thermonuclear plasma. This information is therefore essential to obtain realistic equilibria [1]. These measurements were therefore used in the past for real-time control of the magnetic configuration, and of the q profile in particular [2], but their quality was very often not completely adequate to the objectives of configuration control in JET.

Obtaining the line integrated density from the phase shift is also very appealing particularly for machine protection. Indeed, interferometry, the reference measurement of the electron density in Tokamaks, can be affected by fringe jumps. Once a fringe jump has occurred, the interferometric measurement is compromised for the whole discharge and techniques to overcome the problem have not proved completely satisfactory yet [3]. Obtaining a reliable estimate of the line integrated density from polarimetry would alleviate this problem, since the Faraday rotation and phase shift are not affected by fringe jumps. The importance to provide the line integrated density in real time is very significant, to implement control schemes such as the protection of the wall from shine through of the neutral beam when the density becomes too low.

On JET, a dual interferometer/polarimeter system performs, routinely, the Faraday rotation and Cotton-Mouton phase shift measurements (see Section II). In the case of JET, these measurements are complicated by an "anomalous" phase shift induced by non identified optical components of the diagnostic. The effect of this anomalous instrumental phase shift must be taken into account in the calibration and signal processing procedures. Considering the complex optical paths of polarimetric 
diagnostics on the next generation of devices, such as ITER or Weldenstein $7 \mathrm{X}$, it is expected that similar issues could be encountered. In this perspective, the experience acquired on JET is of unique value since its polarimeter is the only instrument of the same order of complexity in terms of optical components. Moreover, the plasma parameters are close enough to ITER to allow testing solutions for relevant ranges of Faraday rotations and Cotton Mouton phase shifts. For example on JET it is possible to investigate the nonlinear interaction between the Faraday rotation and the Cotton Mouton effect at high currents and densities [4]. Particularly interesting are also the measurements of the horizontal chords, which require particular attention and additional modeling efforts for their interpretation (see Section V).

Since the optical components, introducing the anomalous ellipticity, are completely unknown, the previous algorithms to interpret the calibration data were based on empirical models, in which purely numerical and quite complex fitting procedures were implemented to evaluate the calibration parameters and the polarimetric measurements. This processing method was developed without any investigation on the source of the phase shift anomaly, and it worked as a black box without a clear relation with the hardware setup (for example these calibration procedures did not make any attempt to separate the electronics from the optical instrumental effects). A statistical analysis, carried out on the polarimetric measurements acquired during the campaigns 2003-2009, has highlighted some calibration problems affecting mainly the experimental data of the most recent campaigns (2008-2009), particularly those of the high current discharges. Therefore, it has been decided to develop a new calibration code, separating the electronics from the optical instrumental effects. The electronics calibration constants are determined independently (see Section III), and an independent algorithm models the optical layout of the diagnostic. This new approach resulted in a more transparent calibration code, with better performance in terms of accuracy; moreover the new algorithm is capable of properly modeling the behavior of the diagnostic for all the various configurations, including the ones optimized for high current operation (see Section III and references for more details).

The original version of the new calibration algorithm was developed for offline analysis, but the very positive results obtained have motivated the refinement of the procedures, with the aim of providing well calibrated measurements on a shotto-shot basis mainly for protection and real-time applications. The main difficulty in this respect is the fact that, due to the complex layout of the system (see Fig. 1), the diagnostic is not completely stable. Therefore, the parameters of the model, simulating the optical path, have to be slightly re-adjusted on a shot-to-shot basis to obtain accurate measurements. This is a potential problem also for the next generation of devices, whose diagnostics will probably be even more difficult to access for maintenance and adjustment than on JET. In this perspective, the opportunity to develop a new version of the calibration algorithms to be run automatically before each discharge has been investigated. Indeed, between 14 and $18 \mathrm{~s}$ before the plasma breakdown, an automatic calibration procedure is run for each JET discharge. One of the subjects of the present paper is therefore the description of the new calibration algorithms to interpret the calibration data, capable of providing reliable results in the interval between the end of the calibration procedure and before the beginning of the plasma (see Section IV). For the vertical chords, these new calibration algorithms guarantee good measurements of the Faraday rotation, which can be used directly in equilibrium codes, and of the phase shift, which can immediately provide the line integrated density for any required purpose. On the other hand, for the horizontal chords, no formula was available to directly derive the line integrated density from the polarimetric measurements. New approximations have been therefore devised (see Section V), which guarantee a line integrated measurement of the horizontal chords within 1 fringe of the interferometric values $\left(1.1410^{19} \mathrm{~m}^{-2}\right)$. The importance of reliably providing calibrated polarimetric measurements in real time is illustrated in Section VI by some recent examples, including the magnetic topology obtained with a new realtime equilibrium code developed for JET. Conclusions are the subject of the last Section VII of the paper.

\section{Polarimetry AND Its ImPlementation ON JET}

\section{A. Polarimetric Measurements}

If a linearly polarized electromagnetic wave is sent into a magnetized plasma the following effects occur [5]:

1) Faraday rotation of the polarization plane proportional to the density times the magnetic field component parallel to the direction of the laser beam propagation.

2) Cotton-Mouton phase shift proportional to the density times the square of the magnetic field component perpendicular to the propagation direction.

These effects can be described by the following equations:

$$
\begin{aligned}
\Delta \Psi & \propto \lambda^{2} \int n_{e} B_{\|} d z \\
\Phi & \propto \lambda^{3} \int n_{e} B_{\perp}^{2} d z .
\end{aligned}
$$

In (1) and (2), $\lambda$ is the laser wavelength, $n_{e}$ is the plasma electron density, and $B_{\|}$and $B_{\perp}$ the parallel and perpendicular components of the magnetic field, respectively. To summarize, traversing a magnetized plasma, a polarized beam suffers a rotation of the polarization plane due to Faraday Rotation and acquires ellipticity due to the Cotton-Mouton effect.

There is a major advantage in the plasma density measurements derived from the Cotton-Mouton phase shift with respect to classical interferometry and this is the fact that the measurements are not affected by fringe jumps. Given the importance of careful density control in JET with the new metallic wall, and on a longer term in ITER, having an alternative reliable measurement of the plasma density is extremely valuable.

\section{B. Brief Description of JET Interferometer/Polarimeter}

On JET, the finite-impulse response (FIR) diagnostic operates as a dual interferometer/polarimeter [6], [7]. The system probes the plasma with four vertical and four lateral laser 


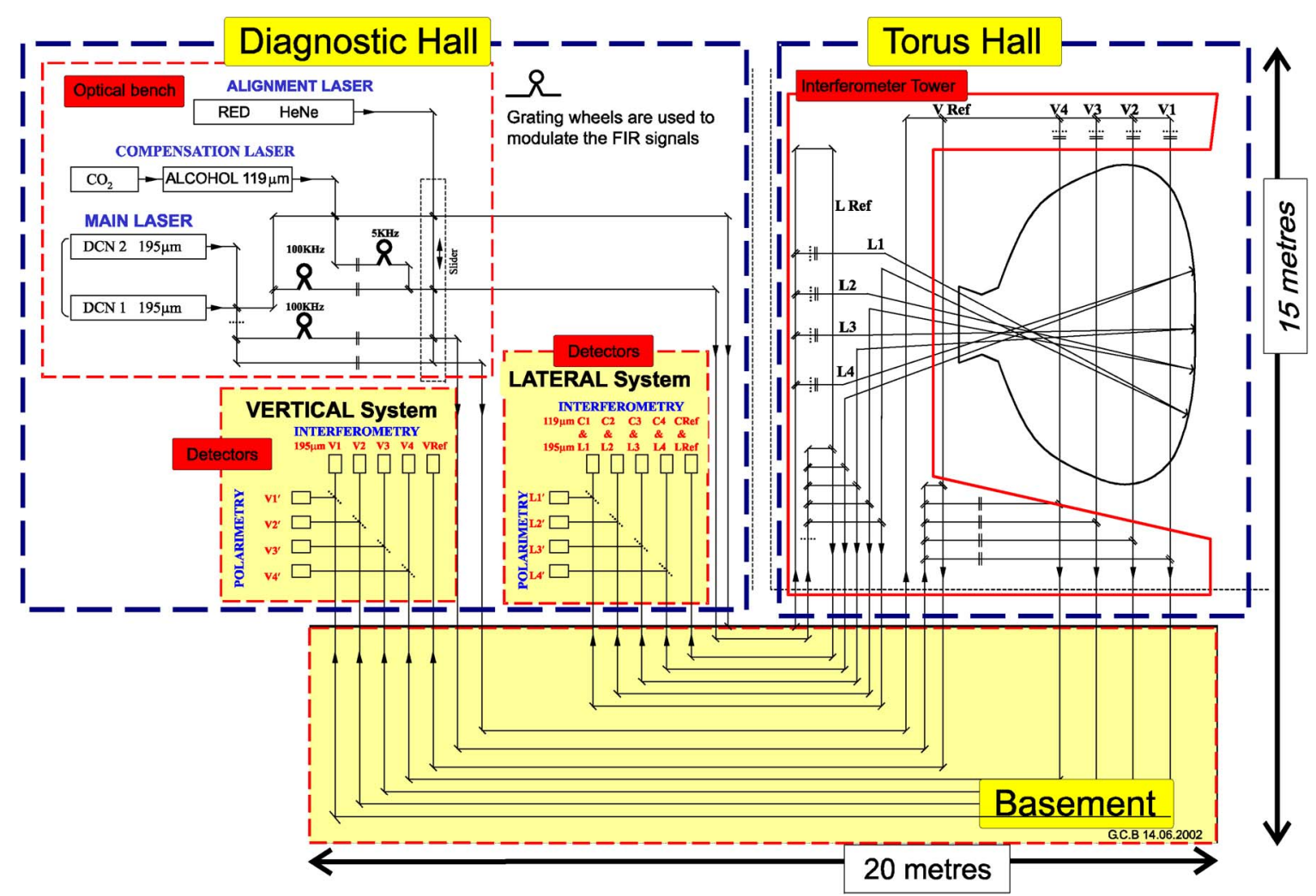

Fig. 1. Schematic of the JET FIR Interferometer/Polarimeter. The vertical channels V1-V4 are referered to as chords 1 to 4 in the text. The lateral channels L1-L4 are referered to as chords 5 to 8 in the text.

beams. The diagnostic provides the line-integrated plasma density measurements, by means of interferometry, and Faraday rotation angle and Cotton-Mouton phase shift measurements by polarimetry. These measurements are preceded by an on-line calibration procedure performed before each shot (using halfwave plates).

The layout of the instrument is shown in Fig. 1.

The main characteristics of the experimental setup are:

- the diagnostic involves the use of far infrared lasers because this is a good compromise between the conflicting needs of increasing the sensitivity of the instrument and of avoiding the cutoff at the plasma frequency [8];

- two FIR lasers (195 $\mu \mathrm{m}$ deuterated cyanide and $119 \mu \mathrm{m}$ Methanol), modulated at two different frequencies, allow vibration-induced and plasma-induced phase shifts to be measured (in the past, only the horizontal chords were equipped with the second color for vibration compensation, but for the next campaigns, a second wavelength will be implemented also on the vertical chords);

- very long optical path: about $80 \mathrm{~m}$ for each of its eight channels;

- the time resolution of $10 \mu \mathrm{s}$;

\section{The Calibration Procedure And the Algorithm to Derive the Calibration Constants}

\section{A. The Calibration Procedure}

At JET, the calibration procedure is the following: the halfwave plate, located at the entrance of the vacuum vessel, is rotated (via a step-motor) of a well-known angle and the phase shift is recorded at each angle, while the Faraday rotation is approximately equal to twice the rotation angle of the halfwave plate. This operation takes place in the interval between $18 \mathrm{~s}$ and $14 \mathrm{~s}$ before the plasma. The $14 \mathrm{~s}$ from the end of the calibration procedure to the plasma breakdown are therefore the time allowed to run the calibration code and to produce the required calibration parameters.

In addition to this routine procedure, before each campaign, or when it is necessary, a full manual calibration is performed. It consists not only of the same steps, as the ones performed before the shots, but also of the fine tuning of the angle of the wire grid, located in front of the two detectors. This allows modifying the repartition of the signal between the interferometric and the polarimetric detectors and therefore permits to optimize the setup of the diagnostic for the requirements of the physical program (depending for example on the relative importance to be accorded to the interferometric and polarimetric signals).

Unfortunately, the value of the phase shift is nonlinear with the half-wave angle, contrary to what would be expected for a system with only ideal optical components. Because of this non ideal behavior, at JET the polarimetric signals are processed using a model which assumes that an unspecified optical element generates an "anomalous ellipticity" characterized by a constant phase shift referred to a rotated co-ordinate system of unknown orientation [7].

\section{B. New Calibration Algorithms}

As mentioned, a new algorithm has been developed to extract more robust calibration parameters, valid over the whole 
range of JET discharges, from the signals acquired during the calibration [9], [10].

In the new code, all optical components, crossed by the laser beam for the vertical chords, have been modeled using the Mueller Matrix formalism [11]. The optical components involved in the model are: the half-wave plate, the wire grid located in front of the detectors, and a cascade of retarders to reproduce the "anomalous ellipticity," which afflicts the polarimetry measurements. A quite comprehensive statistical analysis has been carried out to determine the number of retarders required to model each chord (more details are given in the following sections and in the references). Thus, known the initial state of polarization and the Mueller matrices for all the optical components, it is possible to calculate the polarization angle and the phase shift, given by:

$$
\begin{aligned}
& \psi=\frac{1}{2} \arctan \left(\frac{S_{2}}{S_{1}}\right) \\
& \varphi=\arctan \left(\frac{S_{3}}{S_{2}}\right)
\end{aligned}
$$

where $S_{1}, S_{2}$, and $S_{3}$ are the components of the final Stokes vector $\left(\vec{S}_{1}\right)$.

These parameters are linked to the Faraday rotation angle $(\Psi)$ and to the Cotton-Mouton effect $(\Phi)$ by:

$$
\begin{aligned}
& \Psi=\psi-\Theta_{0} \\
& \Phi=\varphi-\phi_{0}
\end{aligned}
$$

where $\Theta_{0}$ is $45^{0}$ and $\phi_{0}$ is the phase shift between the orthogonal electric field components in absence of plasma. All the details about this method to obtain the calibration constants of JET polarimeter can be found in [10].

In the following Figs. 2 and 3, an example of the fits of the experimental calibration curves obtained with the new calibration code are shown. The polarimetric measurements obtained with this new calibration algorithm in a wide range of plasma parameters have been reported in [10]. A systematic validation of the results has been performed with the method of the autocorrelation of the residuals as documented in [12].

\section{Calibration Algorithms for Shot-to-Shot Adjustments of the Calibration Factors}

The algorithms that interpret the data of the calibration procedure are meant to provide the parameters of the optical components used to model the various chords. In the case of the vertical chords, typically two retarders are sufficient, and, therefore, the unknown parameters, to be determined by the calibration algorithms, are the four angles of the retarders and the angular position of the wire grid. In general, they can assume any value between 1 and 360 and therefore the number of combination is $360^{5}$. Of course, the investigation of all these combinations cannot be performed in the interval before the plasma breakdown. Therefore, a combined approach has been tested for JET. The parameters of the optical model are determined with an exhaustive scan of all the angles after the last manual calibration. Then, on a shot-to-shot basis, the values

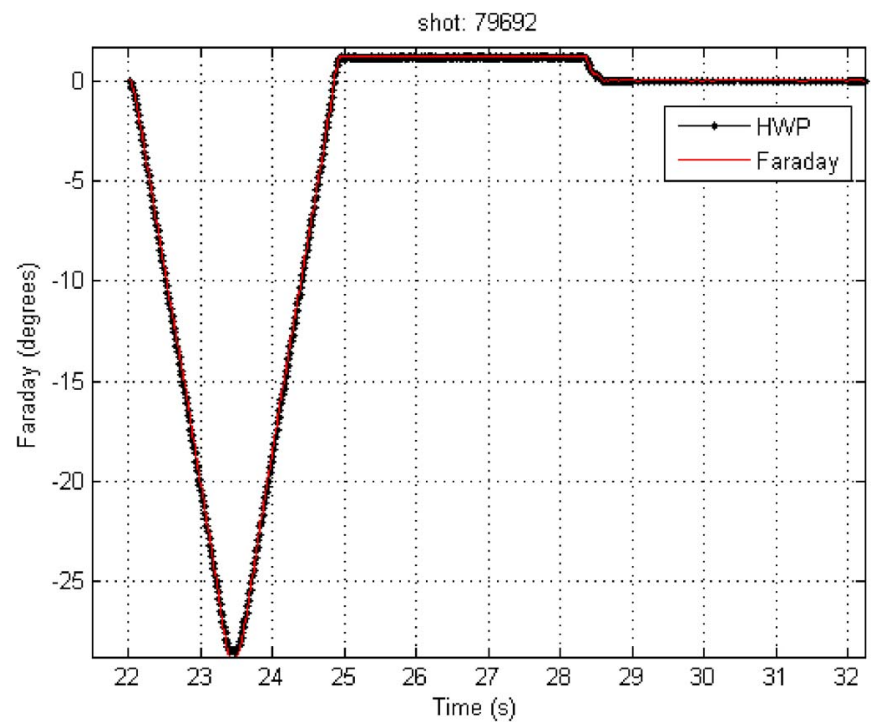

Fig. 2. Comparison between the experimental calibration curve (black dotted line) and the estimate of the new calibration model (red line). Shot: 79692 chord \#3.

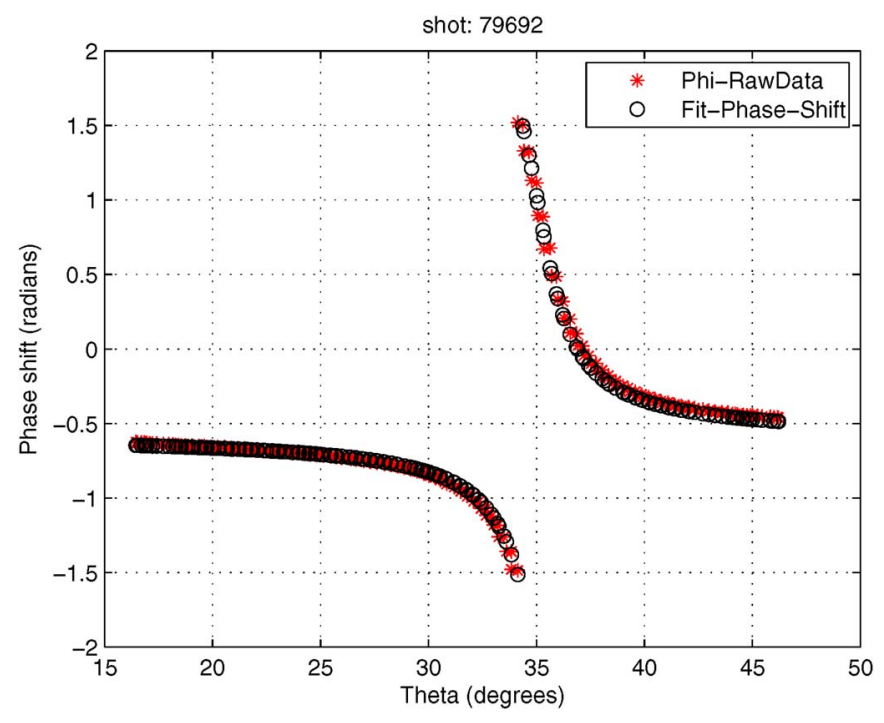

Fig. 3. Phase shift calibration curve (shot:79692 chord \#3). Comparison between the experimental calibration curve (red asterisks) and the estimate of the new calibration model (black circles). On the $\$ x \$$-axis Theta is the rotation angle of the half-wave plate.

of the parameters are adjusted before the plasma breakdown. This is achieved with a limited scan on the various angles with a resolution of $10^{-3}$ degrees. A sensitivity analysis has been performed to determine the appropriate range of angles to be scanned before every shot for each chord. The details will be discussed when the results for each chord are presented in the next sections.

\section{IMPLEMENTATION OF THE SHOT-TO-SHOT Calibration Code: Vertical Chords}

For the vertical chords, the optical components involved in the model are: the half-wave plate, the wire grid located in front of the detectors, and a cascade of two retarders to reproduce the anomalous ellipticity, which afflicts the polarimetry 
measurements. Fixed the angle of the wire grid, the unknown parameters to be found with the calibration code are 4: the phase shift values and the angles at which the two retarders are located.

In this case, the optimization algorithm is able to refine the calibration in the phase of the shot before the plasma breakdown. A systematic investigation has shown that the range of angles to be investigated is $\pm 8^{\circ}$ for the four parameters of the retarders and $\pm 1^{\circ}$ for the wire grid position.

This optimized function needs about $2 \mathrm{~s}$ to find the best values for the 4 unknown parameters, fixed the angle of the wire grid. This time has been estimated using a laptop with the following proprieties:

processor: Intel(R) Core(TM)2 DuoCPU T8100 $2.10 \mathrm{GHz}$; memory (RAM): $4 \mathrm{~Gb}$; operating system: 32-bit

Hence, the time required is substantially shorter than the available time for the calibration. Even if also the angle of the wire grid is varied over a range of $\pm 1^{\circ}$, the computational time remains well below $10 \mathrm{~s}$, which is still compatible with the time available before plasma breakdown.

Once the calibration curves for the Faraday rotation and the Phase shift have been obtained, the experimental signals with plasma can be analyzed. In particular, for the vertical chords, it is possible to evaluate, in real time too, the density from the Cotton-Mouton measurements, because the toroidal field $\left(B_{t}\right)$ is constant along the line of sight, and thus (2) can be rewritten as

$$
\Phi=k \cdot \lambda^{3} \cdot B_{t}^{2} \cdot \int n_{e} \cdot d z .
$$

Therefore, the line-integrated electron density $\left(n_{e}\right)$ can be directly obtained

$$
\int n_{e} \cdot d z=\frac{\Phi}{k \cdot \lambda^{3} \cdot B_{t}^{2}}
$$

whit $\lambda=195 \mu \mathrm{m}$ and $k$, expressed in $\mathrm{rad} / \mathrm{mT}^{2}$, is equal to

$$
k=\frac{e^{4}}{16 \pi \cdot c^{4} m_{e}^{3} \varepsilon_{0}} \cong 2.4 \cdot 10^{-11} .
$$

To test the quality of the new code, a statistical analysis has been carried out. The interferometer density has been used as reference to validate the results obtained with the new calibration. The results reported in the following are particularized for chord three, which is representative of all the vertical chords. A word of caution should be spent for chord \#4, which in some cases requires four instead of two retarders to be properly modeled. This fact does not introduce any conceptual complication and simply requires longer computational times.

The density estimated by the newly calibrated CottonMouton phase shift is typically in very good agreement with the interferometric measurements as shown in Fig. 4 for a recent shot (79790).

Fig. 5 shows the difference in fringes between the interferometer and the polarimeter density $\left(1\right.$ fringe $\left.=1.14 \cdot 10^{19} \mathrm{~m}^{-2}\right)$ for 42 shots (79782-79817). This database comprises shots after a single manual calibration. Therefore, the reference point for the optical model is the same (see Section III-C). For each

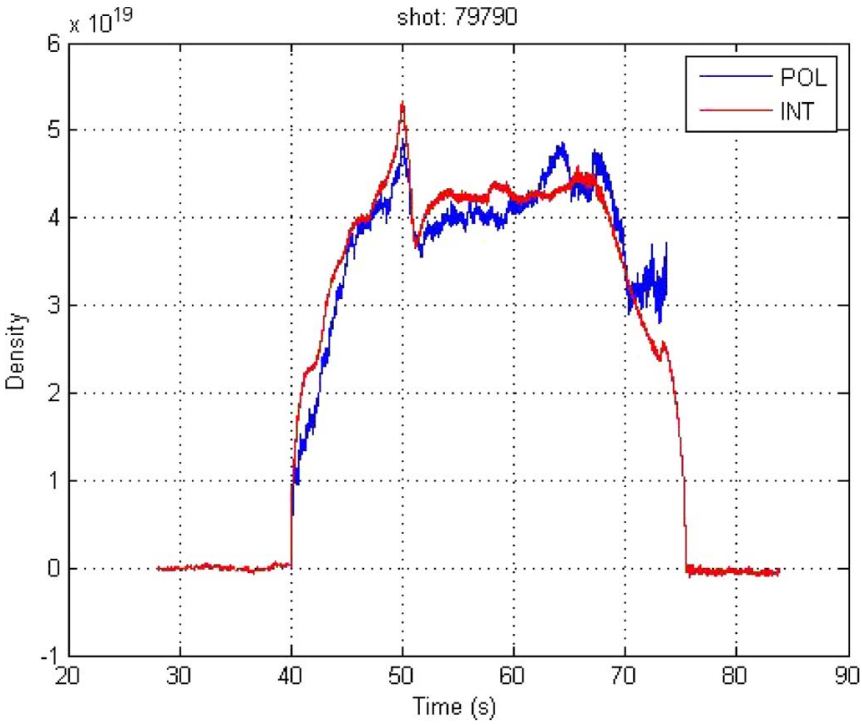

Fig. 4. Comparison between the density with the new calibration (blue line) with the interferometer (red line). Shot: 79790, chord 3.

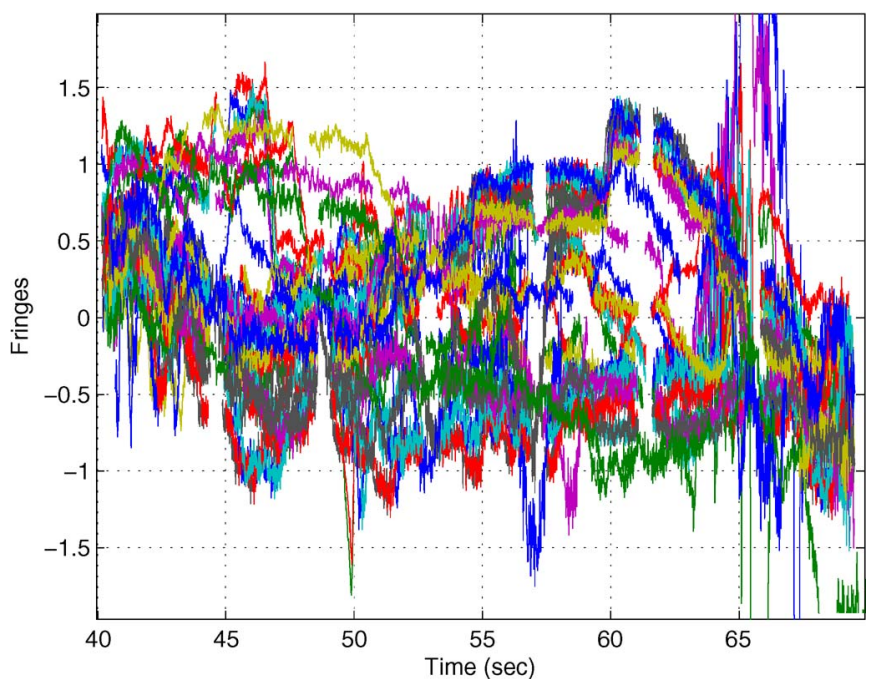

Fig. 5. Difference in fringes between the interferometer and the polarimeter density. Chord 3.

shot, the automatic procedure to adjust the calibration values has been run. As can be seen in Fig. 5, the difference between the interferometer and the polarimeter estimates of the density is always approximately between \pm 1 fringe. It is noteworthy that the points, for which the discrepancy exceeds \pm 1 fringe, are typically at the end of the discharge during the ramp down of the current, when the polarimetric signals are very low and the $S / N$ ratio decreases significantly. The new upgrades of the diagnostics should improve this situation significantly.

In Fig. 6, the absolute value of the polarimeter density is plotted versus the absolute interferometer density. The linear relation between the two is satisfactory. It is also interesting to note that this agreement extends over a range of more than an order of magnitude (from less than $110^{19}$ to more than $10^{20} \mathrm{~m}^{-2}$ ). This is an important fact confirming the quality of the adopted approach. Indeed, as mentioned, the calibration reference point is the same over the entire range of densities, 


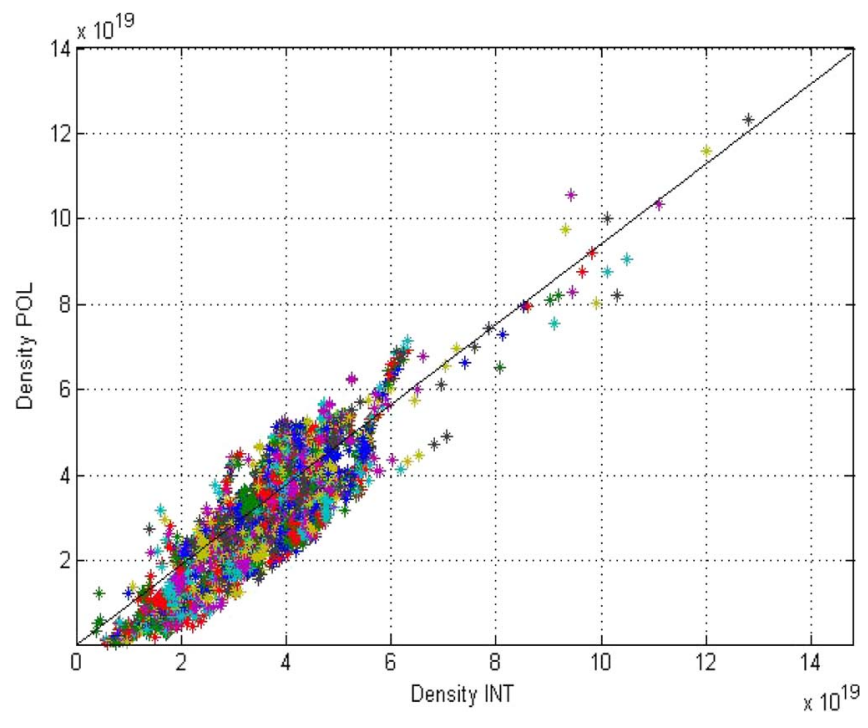

Fig. 6. Polarimeter density versus interferometer density. Chord 3.

proving the potential of the optical model developed. It is also worth mentioning that these results prove the validity of the technique for density values of relevance for ITER.

Another important aspect to note is that, with the developed algorithms, no manual modification is needed to adjust the calibration even over a range of densities exceeding an order of magnitude. Previous calibration techniques required a complete revision of the fitting procedures over intervals of densities a fraction of the one considered in this example. The small variations in the optical parameters, implemented by the automatic refinement procedure, take into account the small variations in the diagnostic settings, which occur between shots and are an unavoidable aspect of an optical diagnostic of the complexity of JET interferometer-polarimeter.

It is worth mentioning that the developed method allows converging on an optical model of the diagnostic, which interprets properly not only the phase shift but also the Faraday rotation. The same procedure provides a unique set of parameters, which provide also a coherent interpretation of the Faraday rotation and not only of the density (see Fig. 7).

\section{IMPLEMENTATION OF THE SHOT-TO-SHOT CALibration Code: Lateral Channels}

Since from a hardware point of view, in the horizontal optical paths, the only main difference is the presence of the in-vessel mirror, which can be easily modeled with a fixed Mueller matrix, the new calibration procedure and the subsequent elaboration of the raw data, to evaluate the Faraday rotation and the phase shift angle, are the same as for the vertical channels [10]. Again, only the parameters of the retarders (the phase shifts $\delta_{i}$ and the angles $\alpha_{i}$ at which the retarders are located) have typically to be determined with a series of scans before the breakdown.

Also, in this case, an optimization function has been developed to refine, on a shot-to-shot basis, the values of the unknown parameters. Scanning the parameters in the same range shown for the vertical channels $\left( \pm 8^{\circ}\right.$ for the four parameters of

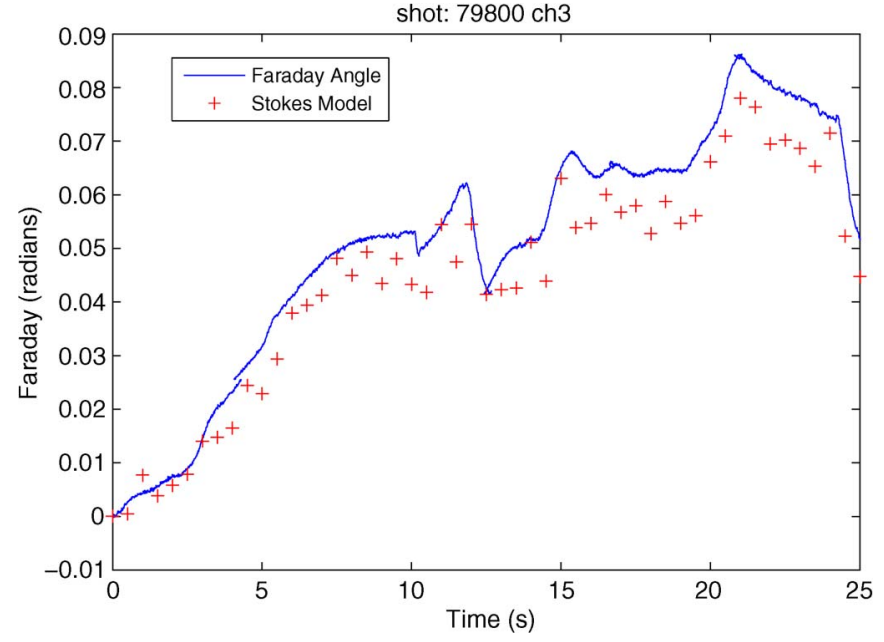

Fig. 7. Comparison of the experimental Faraday rotation obtained with the described calibration procedure and the one calculated by a propagation code implementing the Stokes formalism [10] and using EFIT estimate of the fields for channel 3.

the retarders and $\pm 1^{\circ}$ for the wire grid position) and using the same laptop, the time needed to find the best values is about $2 \mathrm{~s}$.

On the other hand, no simple formula, equivalent to relation (8) for the vertical chords, is available to obtain the density from the polarimetric measurements of the horizontal lines of sight. In JET also, there are no validated propagation codes that perform a systematic comparison between the polarimetric and the interferometric measurements. Therefore, approximate formulas have been devised to benchmark the results obtained with the new calibration code. It is worth mentioning that, since the plasma density is definitely one of the most important physical parameters to be controlled during a plasma pulse, the approximations tested utilize only quantities which are easily available in real time, not only on JET but also on the vast majority of Tokamak devices. The approximations themselves are believed to be equally valid in other devices and not specific to JET.

\section{A. Line Integrated Density in Real-Time Theory and Approximations}

Using the Stokes vector formalism, the Faraday angle and the phase shift can be expressed in the following way [10], [13]:

$$
\begin{aligned}
\tan (2 \psi)= & C_{1} \int_{r_{0}}^{r} n_{e} B_{r} d r \\
\tan (2 \phi) & =\frac{-C_{2} \int_{r_{0}}^{r} n_{e} B_{t} B_{p} d r}{\tan 2 \psi}
\end{aligned}
$$

with $C_{1}=2 \times 10^{-20}$ and $C_{2}=1.794 \times 10^{-22}$ (calculated for the laser wavelength of $\lambda=195 \mu \mathrm{m}$ of JET plarimeter).

Since the objective is to calculate the line integrated density for feedback control, relations (9) and (10) have to be simplified and expressed in terms of quantities which can be provided in real time. To this end, all the various quantities can be taken out 
[SHOT: 79777]
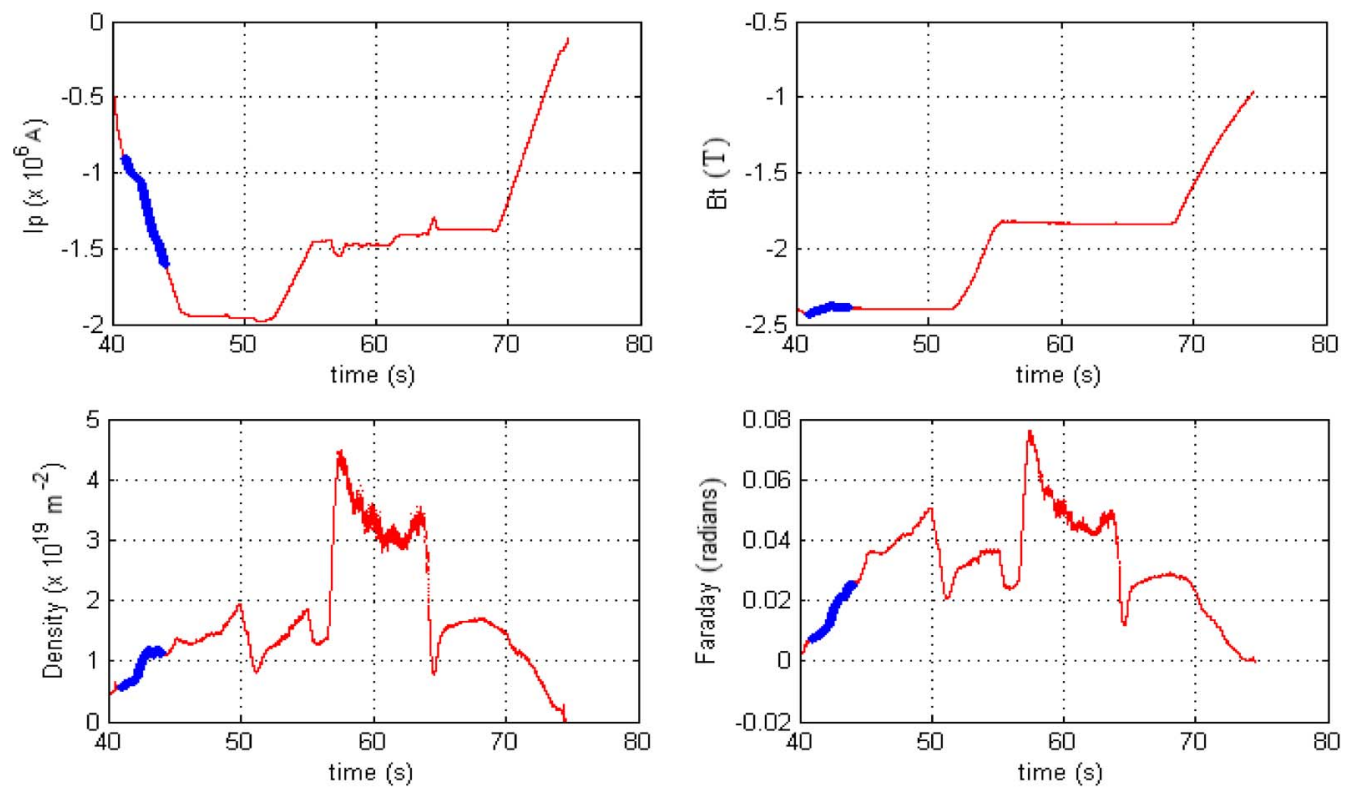

Fig. 8. Variation of the main parameters used in the approximated equation (11) to provide the line integrated density. The blue part of the curves is the interval used to fit the experimental quantities.

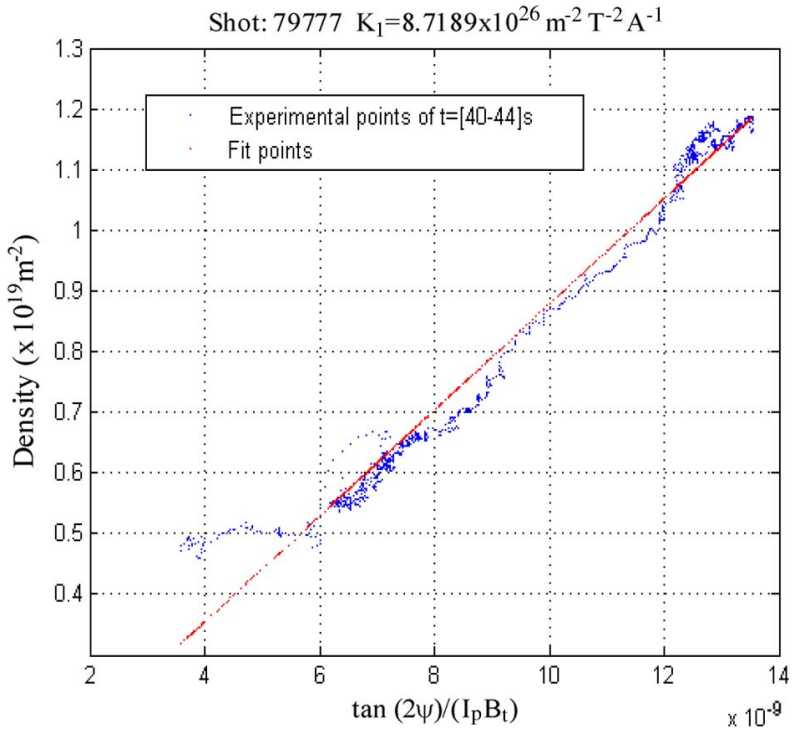

Fig. 9. Determination of the constant K1.

of the integrals (except $n_{\mathrm{e}}$ ), and the profiles effects can be absorbed in an empirical constant. The following approximations for the radial magnetic field $\left(B_{\mathrm{r}}\right)$ and the poloidal magnetic field $\left(B_{\mathrm{p}}\right)$ can be considered:

$$
\begin{aligned}
& B_{r} \propto I_{p} B_{t} \quad \Rightarrow \int_{r_{0}}^{r} n_{e} d r=K_{1} \frac{\tan (2 \psi)}{I_{p} B_{t}} \\
& B_{p} \propto I_{p} \Rightarrow \int_{r_{0}}^{r} n_{e} d r=K_{2} \frac{\tan (2 \psi) \cdot \tan (2 \phi)}{I_{p} B_{t}}
\end{aligned}
$$

where $K_{1}$ and $K_{2}$ are constants that have to be determined empirically.
The $K_{1}$ constant, in (11), is given by the ratio of the integrated density and the term on the right side of relation (11). The constant $K_{1}$ is therefore just the angular coefficient $m$ of the linear equation

$$
y=m x \quad \text { where } \quad\left\{\begin{array}{l}
y=\int_{r_{0}}^{r} n_{e} d r \\
m=K_{1} \\
x=\frac{\tan (2 \psi)}{I_{p} B_{t}} .
\end{array}\right.
$$

For each pulse, the range of times between $40 \mathrm{~s}$ and $44 \mathrm{~s}$, when the gradient of the physical parameters are higher, is scanned and then $K_{1}$ calculated by a linear fit of these points. The chosen range of times is due to the fact that, during the rise of the plasma current, a wider range of angles is scanned, whereas during the flat top, the Faraday rotation remains more constant. Therefore, the beginning of the discharge provides better data for a fit of the constant $K_{1}$. Moreover, obtaining $K_{1}$ during the first seconds of the shot would give the opportunity to implement a shot-to-shot estimate of the constant $K_{1}$. It is indeed possible to conceive to use the value of $K_{1}$ determined for the previous shot during the rise of the plasma current and then update the value of the constant with the new fit obtained during the rise of the current.

The same approach has been used to evaluate the constant $K_{2}$ in (12).

Since we want to obtain general empirical constants to be used in real time to get the integral density using the above expressions, $K_{1}$ and $K_{2}$ should be calculated using a large database of shots. This has been possible only for the constant $K_{1}$, since the signal of the phase shift is typically very low for the horizontal chords. Hence, $K_{2}$ has been preliminarily estimated only for five shots with a sufficiently robust signal of the Cotton Mouton phase shift. The results reported in this 

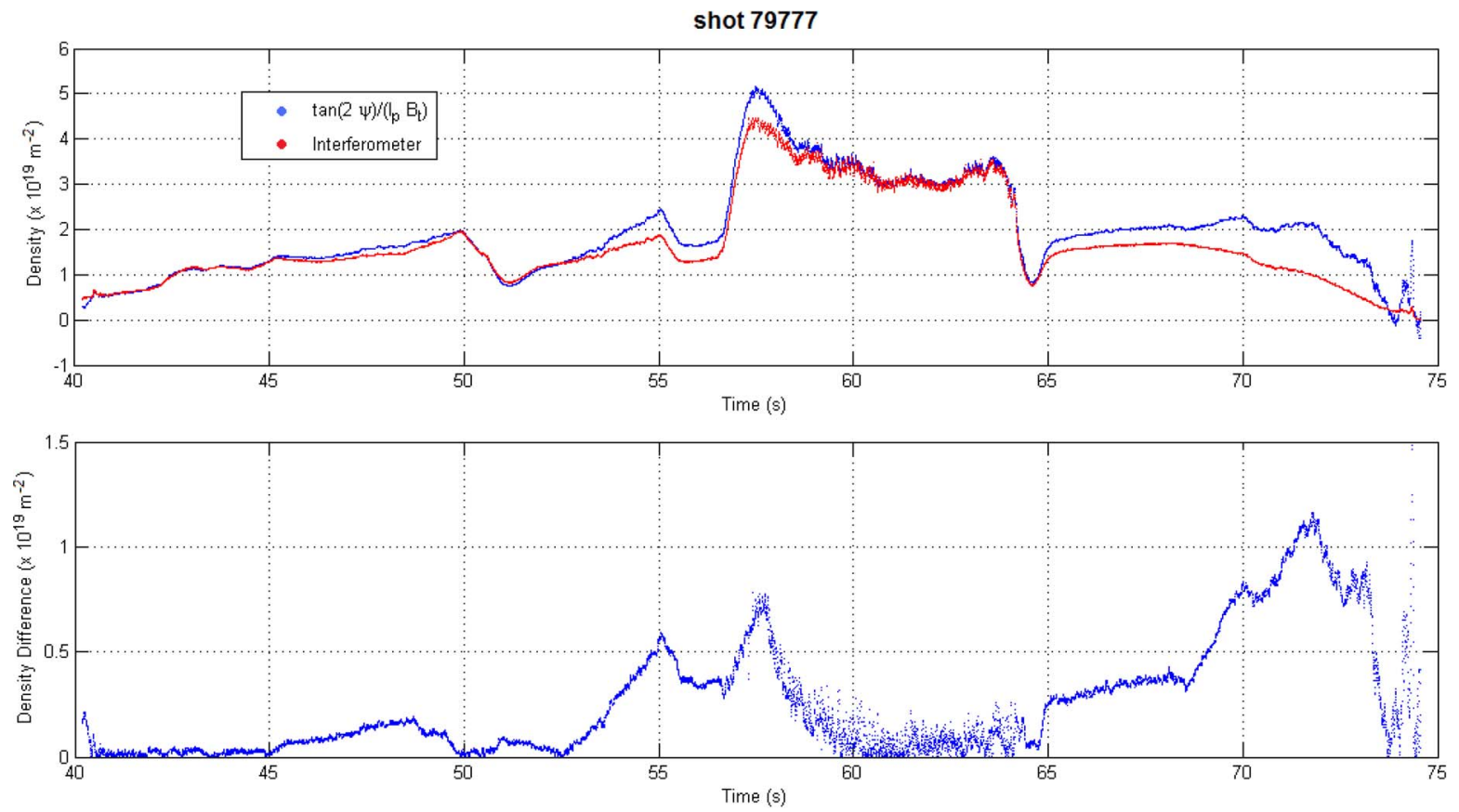

Fig. 10. Comparison of the interferometric density and the density calculated on the basis of equation (11). In the bottom plot the difference between the two estimates is reported.

section are particularized for chord 5, which is representative of the horizontal lines of sight.

\section{B. Line Integrated Density for Channel 5: First Approximation}

In Fig. 8, an example of the range of times considered (blue line) for the determination of the two empirical constants $K_{1}$ and $K_{2}$ is shown.

In Fig. 9, the fit obtained for the $K_{1}$ constant from the range of times considered, for the same pulse as in Fig. 8, is shown.

In Fig. 10, the line integrated density, calculated with the $K_{1}$ value, is compared to the interferometer density.

The calculation has been performed for 27 shots, the obtained values have been averaged obtaining a mean value of the constant $\left\langle K_{1}\right\rangle$ :

$$
\left\langle K_{1}\right\rangle=(9.4 \pm 1.3) \cdot 10^{26} m^{-2} T^{-2} A^{-1}
$$

Using this value in the expression (11), the integral density has been calculated for the same 27 shots (in the range from 68698 to 79853), together with the modulus of the difference between the obtained values and the interferometer line integrated densities. The results are shown in the following Fig. 11.

As it can be seen in the previous Fig. 11, the line integrated density obtained with the present approximation differs from the interferometric measurements typically significantly less than a fringe. Therefore, we can say there is a quite good agreement between the two density estimates particularly for real-time applications. Again, the bigger discrepancies occur at the end of the discharge, when the polarimetric signals are very low.

In Fig. 12, it can be seen how this level of accuracy is maintained for the entire range of JET densities, since the shots analyzed cover about an order of magnitude range of densities.

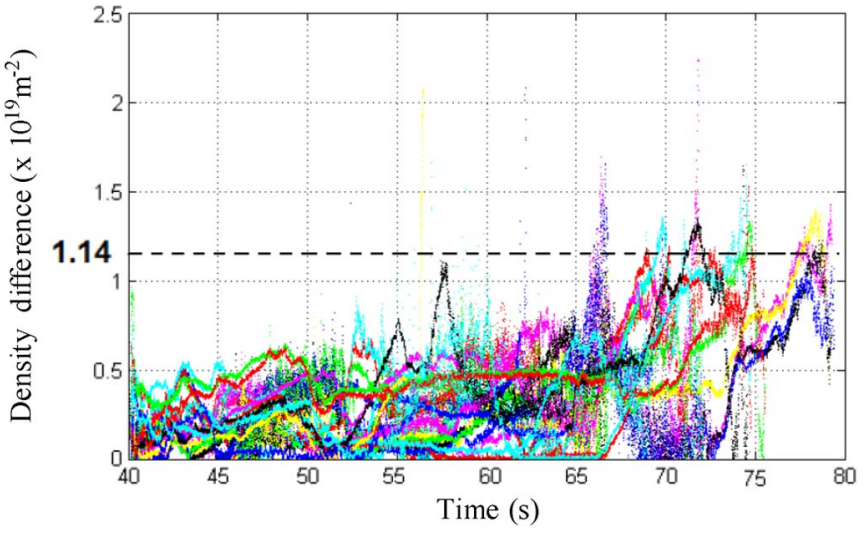

Fig. 11. Statistical comparison of the interferometric density and the density calculated on the basis of equation (11).

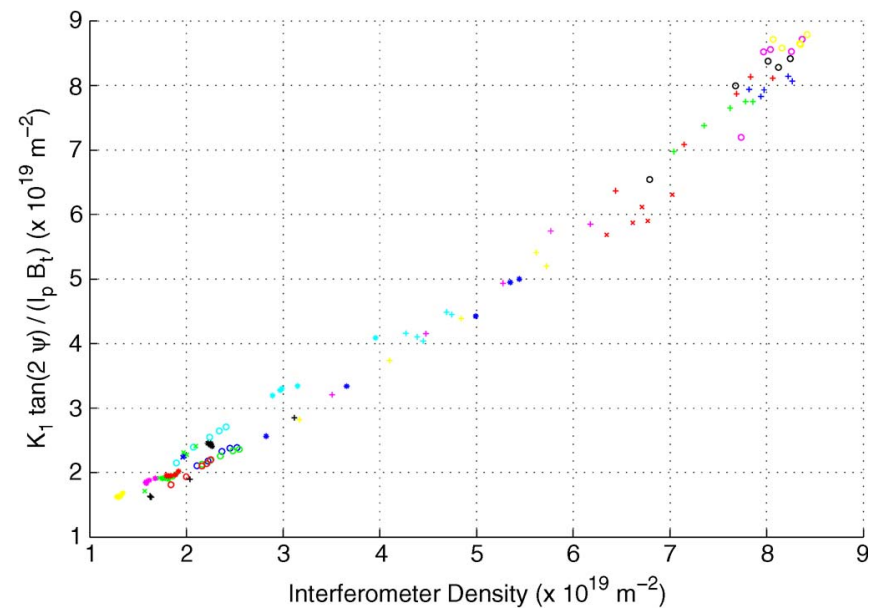

Fig. 12. Polarimetric density estimated with equation (11) versus the interferometric measurements. 


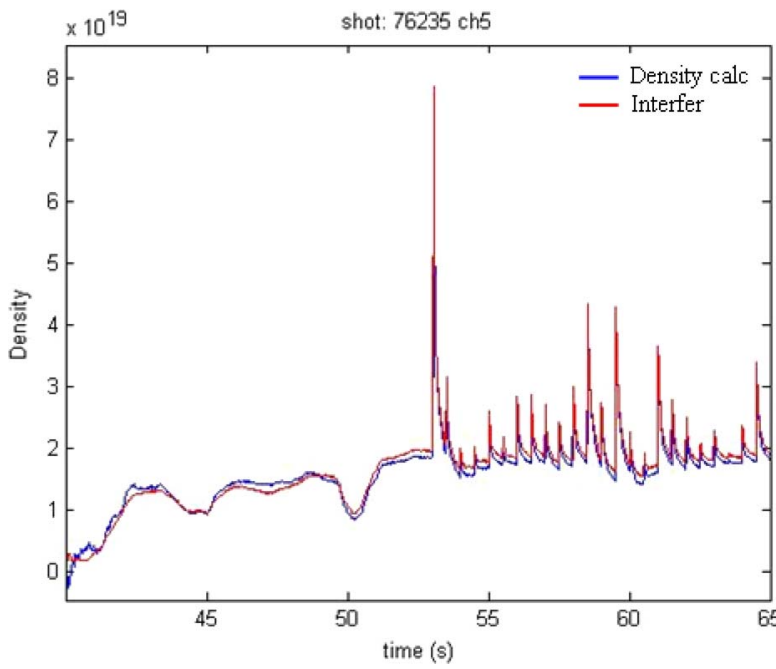

Fig. 13. Polarimetric density, estimated with equation (11), in blue and interferometric density in red for a discharge with a train of different size pellets from 53 to $65 \mathrm{~ms}$.

Even if (11) is a quite rough approximation, it seems to work exceptionally well also in difficult situations, for example when there are strong variations in the density profile during a discharge. A quite extreme example is shown in Fig. 13, in which the estimate provided by (11) is compared with the interferometric density for a discharge with a train of large pellets. The approximate solutions manages to reproduce quite accurately all the density peaks, even if they are known to introduce significant perturbations in the density profiles.

\section{Line Integrated Density for Channel 5: Second Approximation}

In Figs. 14 and 15, the quality of the second approximation ((12)), for the only five shots in which good signals were available together with a proper calibration, is shown.

This first study shows that also the phase shift, provided by the Polarimeter of the JET tokamak, can be used to calculate the plasma line integrated density in real time with an accuracy of better than one fringe.

While it has been possible to make a quite satisfactory statistical validation of expression (11) involving only Faradayeffect data, expression (12) has been just verified only for the five shots with reliable Cotton-Mouton effect signals. On the other hand, given the more solid theoretical background of relation (12), it would be nice to test and implement it because it is expected to provide better results. If the upgrade of the diagnostic, presently being implemented, will provide the expected improvements in the signal to noise ratio of the phase shift measurement for the horizontal chords, approximation (12) will be tested systematically.

\section{ApPlications of the POlarimetric MEASUREMENTS IN REAL TIME}

The availability in real time of reliable polarimetric measurements opens new prospects to the feedback control of Tokamak plasmas. For the purpose of machine protection, the measurement of the line integrated density without fringe jumps from the cotton Mouton effect is a quite important improvement. The control of density is expected to become significantly more reliable and the exploitation of the machine more efficient, since discharges will not have to be terminated simply for the unavailability of the interferometric measurement. The approximations, providing the line integrated density along the horizontal lines of sight, are potentially a very beneficial step forward. In the case of the protection of the wall against the neutral beam shine through, for example, this upgrade is considered particularly relevant now that JET will have to operate with a full metallic wall. It is interesting to underline the usefulness of expression (11) in this respect, since it provides a reliable signal without the drawback of fringe jumps using only quantities already available in real time. This can be seen, for instance, in Fig. 16.

In general the magnetic reconstruction will benefit from the Faraday rotation measurements. Indeed, it has already demonstrated with a systematic analysis that internal measurements of the fields, either with MSE or polarimetry, are essential in a Tokamak to determine the proper equilibrium, since the external magnetic coils alone are not enough to identify the right solution [1]. In the last years, it has become also quite evident that the constraints of polarimetry on the equilibrium can have a significant effect on the determination of the plasma boundary [14]. In this respect, an important example is the following comparison of the various topological parameters, at the edge and in the core of the plasma, obtained with EFIT using the magnetic pickup coils only and with EFTF, the version of EFIT using also the polarimetric measurements of chords 2, 3, 5 and 7. To summarize the results of the comparison, the attention has been focused on the parameter ROG, which measures the plasma wall distance in the outer equatorial plane, the coordinates $Z_{\mathrm{mag}}$ and $R_{\mathrm{mag}}$ of the magnetic axis and the value of the safety factor $q_{95}$ at $95 \%$ of the plasma radius. In Fig. 17, the time evolution of these quantities is shown for a well diagnosed discharge. The estimates of the geometrical quantities by EFTF can differ even of a couple of centimeters, with respect to the values provided by EFIT with the magnetic only. This discrepancy is quite significant and the fact that the EFTF measurements are more accurate has been verified using other diagnostics such as the cameras looking at the plasma edge. In general, including the polarimetric measurements produces more shrunken equilibria, due to the fact that the new constraints force EFIT to locate more current close to the separatrix. The edge safety factor can also be significantly different between the two estimates (see also later).

With regard to the more general problem of profile control, polarimetry has also been the reference internal measurement for advance experiments such as the control of the q profile [2]. In order to obtain the internal magnetic topology in real time, a new equilibrium code has been developed for JET explicitly for this purpose. This new code is called EQUINOX and has been designed and implemented in $\mathrm{C}++$ using a finite-element method and a nonlinear fixed point algorithm associated to a least-square optimization procedure [15]. The code relies on 


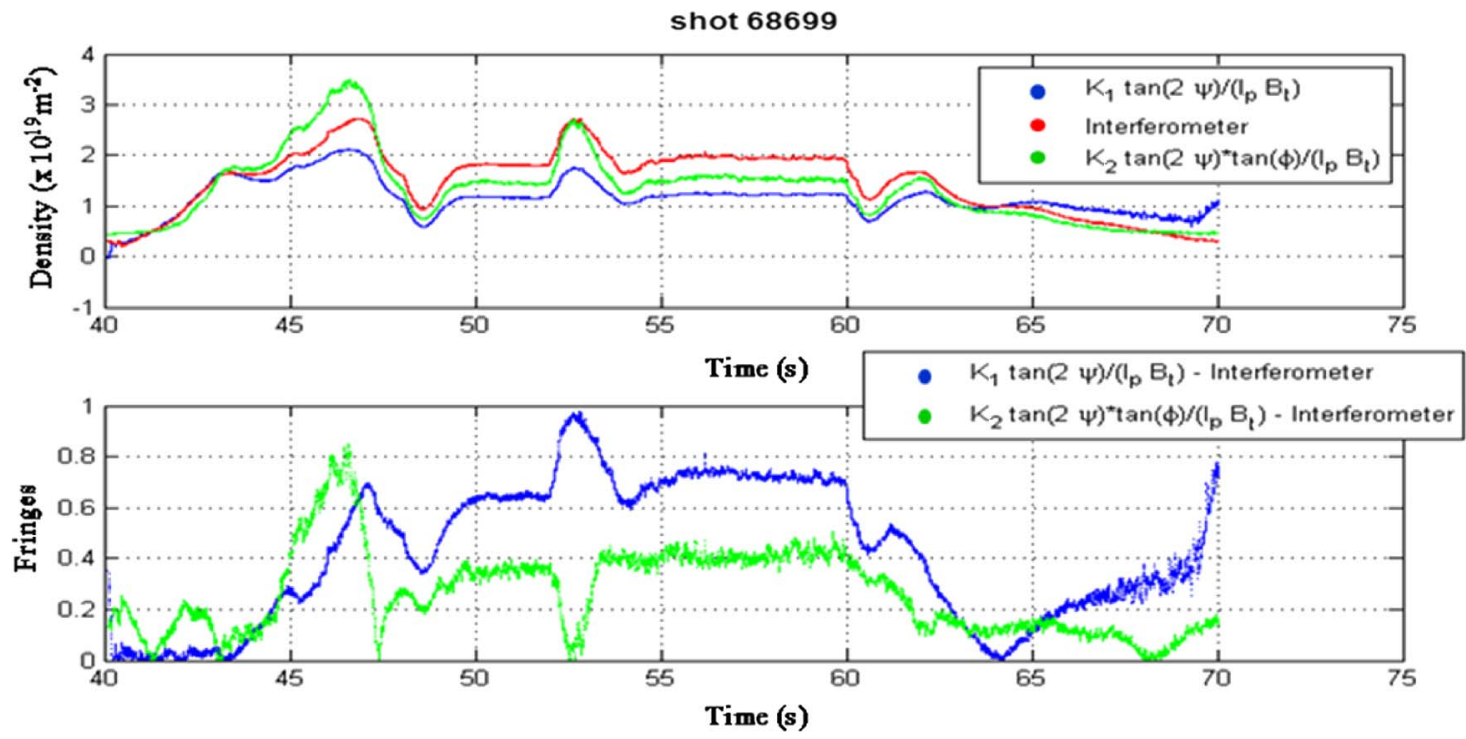

Fig. 14. Comparison of the interferometric density and the density calculated on the basis of equation (12) for shot 70022 . In the bottom plot the difference between the two estimates.

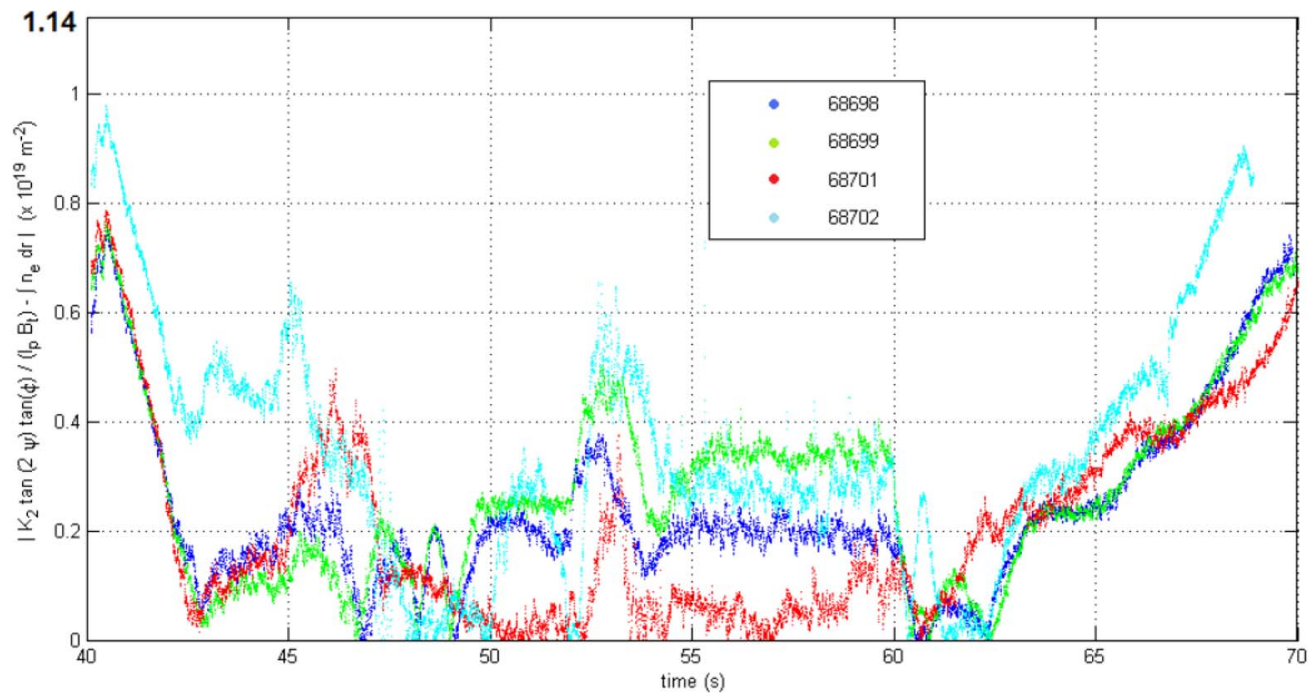

Fig. 15. Statistical comparison of the interferometric density and the density calculated on the basis of equation (12). The accuracy of this approximation is well within one fringe $\left(1.1410^{19} \mathrm{~m}^{-2}\right)$.

tokamak specific software to provide flux values on the first wall of the vacuum vessel. By means of least-square minimization of the difference between the experimental measurements and the simulated ones, the code identifies the source term of the nonlinear Grad-Shafranov equation. The experimental measurements that enable the identification are the magnetics on the vacuum vessel, the interferometric and polarimetric measurements on several chords, and the motional Stark effect measurements. Since the algorithm is initialized from the equilibrium at the previous time step, two or three point iterations are usually enough to ensure convergence. This leads to a very efficient, quick, and robust algorithm.

Recently, the code has been systematically validated and it is ready to run in the framework of JET real-time system. An example of the importance of the magnetic internal measurements to converge on the equilibrium is shown in Fig. 18, in which the
EQUINOX estimates are also compared with the established EFIT reconstructions.

Fig. 18 proves very clearly that the constraints provided by the polarimetric measurements are essential to obtain realistic current distributions at least for nonmonotonic q profiles. This result is also a first benchmark of EQUINOX with the polarimetric measurements. A systematic validation of EQUINOX with internal constraints has been performed and will be reported in a different paper.

\section{CONCLUSION}

The increasing sophistication of the control strategies for both plasma protection and physics experiments in JET has motivated a revision of the calibration procedures of the polarimeter. The aim is to provide accurate calibration constants 


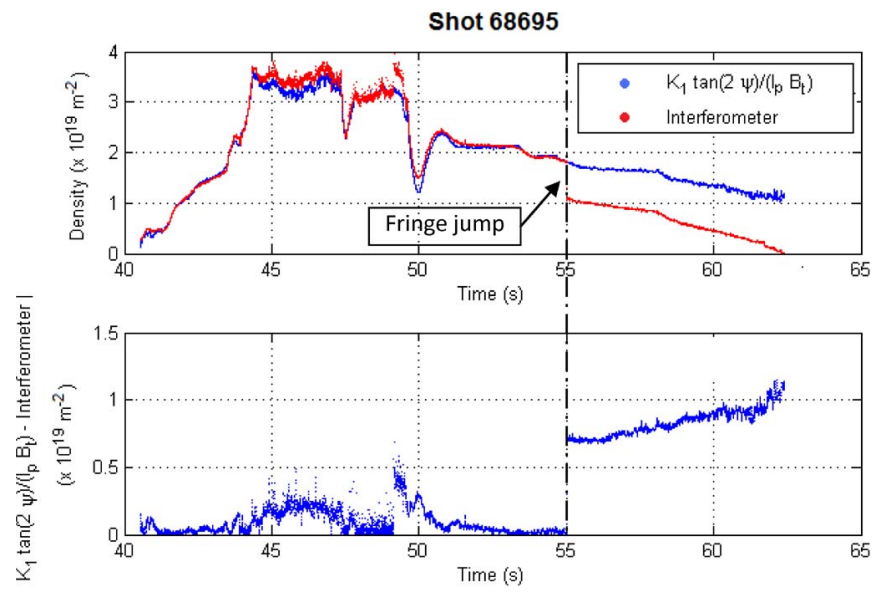

Fig. 16. Comparison of the interferometric density and the density calculated on the basis of equation (11). The robustness of the polarimeric signal is clear whereas the interferometer presents a fringe jump.
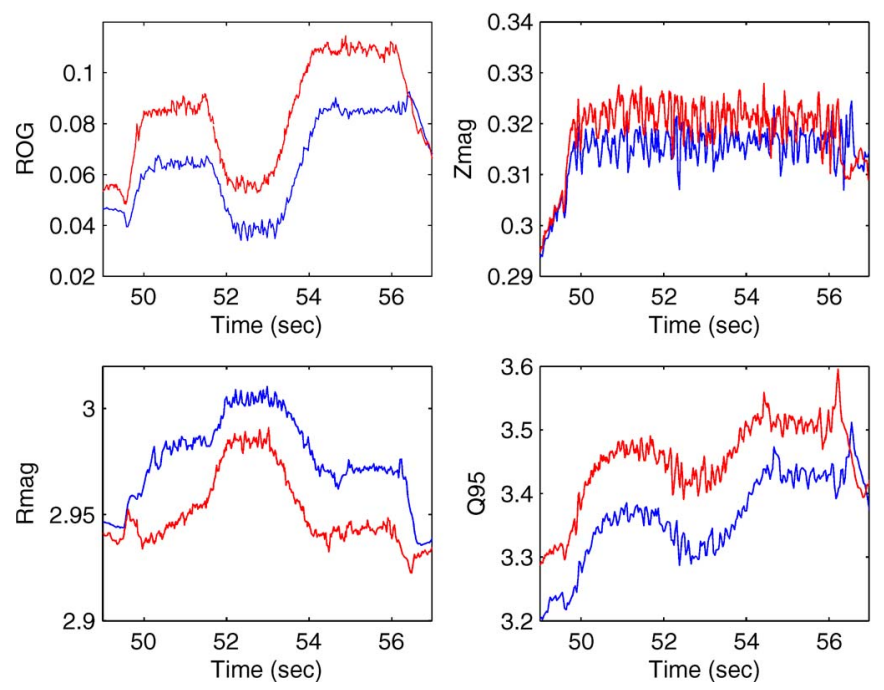

Fig. 17. Comparison of the plasma geometrical parameters and the edge safety factor obtained with EFIT (blue line) and with EFTF (red line) for shot: $77566 . Z_{\mathrm{mag}}$ is the vertical coordinate of the magnetic axis, $R_{\mathrm{mag}}$ the horizontal coordinate of the magnetic axis, ROG the plasma wall distance in the outer equatorial plane and $q_{95}$ the safety factor at $95 \%$ of the plasma radius.

in the interval between the beginning of the on line calibration and the plasma breakdown. This approach has the potential to provide accurate polarimetric measurements in real time on a shot-to-shot basis for the operational space of JET.

To this end, a new calibration algorithm has been refined and optimized, to interpret the calibration measurements in the time interval allowed for the data processing before a discharge. For the vertical chords, the results obtained for the line integrated density, based on the well-known formula for the CottonMouton effect, are within one fringe of the reference interferometer measurements. The estimates of the Faraday rotation have been successfully verified with the most used propagation code available. In order to validate the horizontal chords, new approximate formulas have been derived, which provide the line integrated density from the Faraday rotation and the phase shit measurements. The estimates of these approximated formulas are more than satisfactory, since they provide an

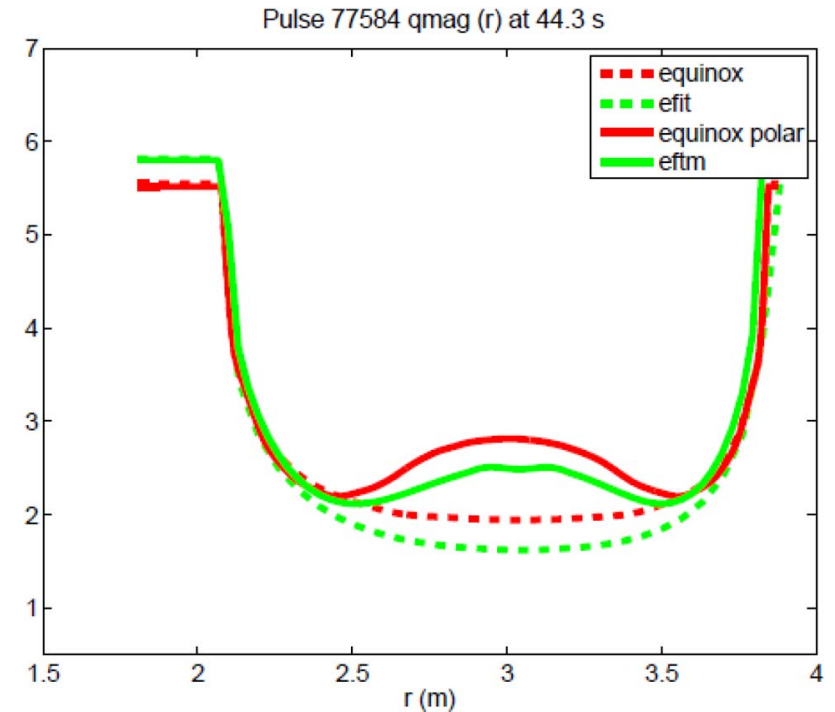

Fig. 18. Comparison of the q profile generated by EQUINOX and EFIT with and without the polarimetric measurements. The dashed curves are the ones obtained without the polarimetric measurements, and the continuous ones have been obtained with the polarimetric measurements.

estimate of the line integrated density within one fringe of the interferometric density for the whole operational range of JET. These results open new perspectives for the use of polarimetry in real time also for machine protection purposes, such as the control of the neutral beam shine through. With regard to more scientific plasma control experiments, the new shot-toshot calibrated polarimetric measurements are planned to be used extensively as constraints for the code EQUINOX. This code implements a solution of the Grad-Shafranov equation providing the entire topology of the magnetic fields in times compatible with the time requirements for the control of the current profile. The version of the code using the polarimetric measurements has already been validated and is ready to be deployed in real time during the next campaigns.

\section{REFERENCES}

[1] F. S. Zaitsev, D.-P. Kostomarov, and E. P. Suchkov, "Existence of substantially different solutions in an inverse problem of plasma equilibrium reconstruction," presented at the 35th Plasma Physics Conference, Hersonissos, Greece, 2008, P-1-091.

[2] L. Laborde, D. Mazon, D. Moreau, A. Murari, R. Felton, L. Zabeo, R. Albanese, M. Ariola, J. Bucalossi, F. Crisanti, M. de Baar, G. de Tommasi, P. de Vries, E. Joffrin, M. Lennholm, X. Litaudon, A. Pironti, T. Tala, and A. Tuccillo, "A model-based technique for integrated realtime profile control in the JET tokamak," Plasma Phys. Control. Fusion, vol. 47, no. 1, pp. 155-183, Dec. 2004.

[3] A. Murari, L. Zabeo, A. Boboc, D. Mazon, and M. Riva, "Real time recovery of the electron density from interferometric measurements affected by fringe jumps," Rev. Sci. Instrum., vol. 77, no. 7, pp. 073505-1-073505-9, Jul. 2006.

[4] F. P. Orsitto, A. Boboc, P. Gaudio, M. Gelfusa, E. Giovannozzi, C. Mazzotta, and A. Murari, "Analysis of Faraday rotation in JET polarimetric measurements," Plasma Phys. Control. Fusion, vol. 53, no. 3, pp. 035001-035020, Jan. 2011.

[5] F. De Marco and S. E. Segre, "The evolution of polarization in an inhomogeneous birefringent, optically active medium," Opt. Commun., vol. 23, no. 1, pp. 125-127, Oct. 1977.

[6] K. Guenther, "Complete far-infrared polarimetry measurements at JET," presented at the Proceedings of the 31st EPS, London, U.K., 2004, P5-172. 
[7] K. Guenther, "Approximate method to extract the pure Faraday and Cotton-Mouton effects from polarimetry measurements in a tokamak," Plasma Phys. Control. Fusion, vol. 46, no. 9, pp. 1423-1441, Jul. 2004.

[8] D. Véron, "Submillimeter interferometry of high-density plasmas," in Infrared and Millimeter Waves, vol. 2, Instrumentation, K. J. Button, Ed. New York: Academic, 1979.

[9] M. Gelfusa, M. Brombin, P. Gaudio, A. Boboc, A. Murari, and F. P. Orsitto, "Modelling of the signal processing electronics of JET interferometer-polarimeter," Nucl. Instrum. Methods Phys. Res. A, vol. 623 , no. 2, pp. 660-663, Nov. 2010.

[10] M. Gelfusa, A. Murari, P. Gaudio, A. Boboc, M. Brombin, F. P. Orsitto, and E. Giovannozzi, "A new calibration code for JET polarimeter," Rev. Sci. Instrum., vol. 81, no. 5, p. 053507, May 2010.

[11] M. Born and E. Wolf, Principles of Optics. Oxford, U.K.: Pergamon, 1980.

[12] M. Gelfusa, A. Murari, D. Patanè, P. Gaudio, and A. Boboc, "Validation of JET polarimetric measurements with residual analysis," Meas. Sci. Technol., vol. 21, no. 11, p. 115704, Oct. 2010.

[13] S. E. Segre and V. Zanza, "High-frequency polarimetric measurement of both electron density and poloidal magnetic field in Tokamaks," Plasma Phys. Control. Fusion, vol. 46, no. 7, pp. 1087-1094, May 2004.

[14] M. Brix, N. C. Hawkes, A. Boboc, V. Drozdov, and S. E. Sharapov, "Accuracy of EFIT equilibrium reconstruction with internal diagnostic information at JET," Rev. Sci. Instrum., vol. 79, no. 10, p. 10F325, Oct. 2008.

[15] J. Blum, C. Boulbe, and Faugeras B., "Real-time plasma equilibrium reconstruction in a Tokamak," J. Phys. Conf. Ser., vol. 135, no. 1, p. 012019, Nov. 2008.

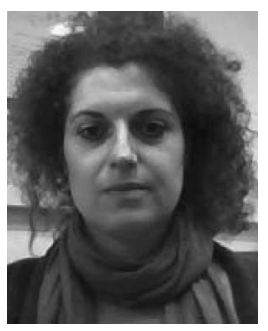

Michela Gelfusa received the B.A. degree in energy engineering and the Ph.D. degree in quantum electronic and plasma physics from the University of Rome "Tor Vergata," Rome, Italy, in 2003 and 2009, respectively. In 2005, she received the M.S. degree in organization and management of companies and health services from the Università Cattolica del Sacro Cuore, Milano, Italy.

Her research interests have been mainly in the field of environmental physics, involving both civil and military applications. In the framework of the collaboration between the University of Tor Vergata and the Joint European Torus she has been involved in various diagnostics, in particular polarimetry, and in advanced data analysis methods.

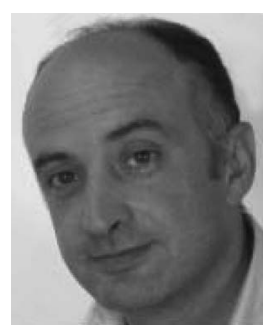

Pasquale Gaudio received the Ph.D. degree in quantum electronics and plasma physic with experimental works on develop and application of X-ray laser plasma system.

Now he is research at University of Rome "Tor Vergata," Rome, Italy. He is expert in laser application and laser diagnostics. He developed and improved a Lidar/Dial systems at the aim of water vapour cycle and columnar atmospheric contents monitoring. He also studied aerosol plume emitted by forest fire and its interaction with laser beams at the aim to study the possibility to use the Lidar/Dial systems to detect forest fire and reduce the false alarm. He collaborates with the Associazione EURATOMENEA Frascati-Fusion Department at the aim of studying the dust mobilization problem inside thenuclear fusion reactors. Inside the same collaboration he is analyzing and interpretate Polarimeter signal at JET fusion reactor in Culham (UK). He is author of more than 100 papers published on International Journal, Conferences Proceedings and two Italian Patent.

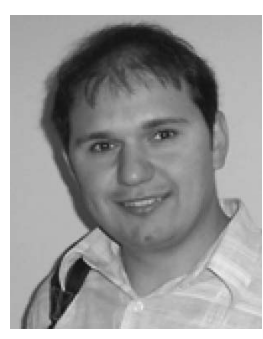

Alexandru Boboc was born in Campulung, Romania, on January 5, 1974. He received the B.A. degree in physics from the University of Craiova Craiova, Romania, in 1997, the M.S. degree in nuclear fusion plasma engineering and the Ph.D. degree in energetics engineering from the University of Padua, Padova, Italy, Italy, in 1999 and 2002, respectively.

During 2002-2004, he worked as Marie Curie post-doc physicist fellow in the nanotechnology domain of magnetic nanonetworks at the University of Limerick, Ireland. Since 2004, he is in charge of the JET Far Infrared interferometer-polarimeter diagnostic system, one of the largest plasma diagnostics systems in the world at present. His main field is the laser based diagnostics systems for nuclear fusion plasmas as well as scientific software programming. He participated actively to the design, installation and operation of various Far Infrared laser-based diagnostic systems on several European fusion experiments as well involvement in the design of the ITER LIDAR diagnostic system.

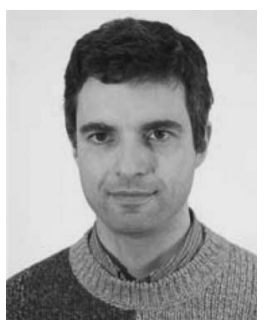

Andrea Murari was born in Verona, Italy, on August 19, 1963. He received the B.A. degree in applied electronics, the M.S. degree in plasma engineering, and the Ph.D. degree in nuclear power plants from the Faculty of Electrical Engineering, University of Padova, Padova, Italy, in 1989, 1991, and 1993, respectively.

$\mathrm{He}$ has mainly worked in the field of measurements for nuclear fusion experiments. He has installed various diagnostic systems on several European experiments, and between 1998 and 2002, he was responsible for the support to all the diagnostics of the RFX experiment. Between 2002 and 2010, he has been the Task Force Leader for Diagnostics (TFD) at the Joint European Torus (JET). Then, since 2003, he has been a Member of the EIROforum Working Group on measurements. Moreover, since January 2008, he has been the Cochair of the European Fusion Development Agreement Topical Group on Diagnostics, becoming chair in 2010. Since 2008, he is also the Coordinator of the European Delegation to the International Tokamak Program Agreement Topical Group on Diagnostics. In 2008, he was nominated as the Group Leader of Diagnostics Control and Data Acquisition in JET. He is currently with Consorzio RFX, Associazione EURATOM-ENEA sulla Fusione, Padova.

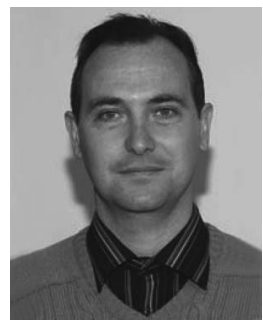

Didier Mazon is a Leader of the EFDA 'Feedback Control Group' in Garching since 2008 and EFDA Deputy Leader of the 'Topical Group Diagnostics' since 2009. Between 2007 and 2010, he was Task Force Leader for Diagnostics on JET. His main duties are to coordinate the control activity in Europe in preparation of ITER (experimental Fusion machine under construction in Cadarache France) and DEMO (project of a Fusion reactor that will follow the ITER experimentation) but also to follow and coordinate the diagnostics activities in the European Tokamaks. In 1999, he joined the CEA Cadarache (France), where he is responsible for the Bremsstrahlung emission and Soft X-rays diagnostics. He works on the development of real-time diagnostics and feedback algorithms for the simultaneous control of the current density and pressure profiles with direct applications in JET and Tore Supra. His research interests include plasma physics, in particular, understanding and control of internal transport barriers, control systems, plasma equilibrium and transport studies. He wrote two patents related to the development of new techniques for the acquisition and unfolding of the Hard X-ray measurement. He published more than 200 articles in 10 years in renowned international journal and conferences as lead author or coauthor. 


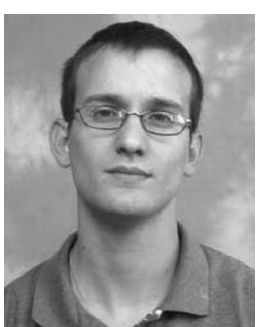

Fabio Avino was born in Rome, Italy, on June 21, 1986. He received the B.A. degree in physics at the University of Rome "La Sapienza," Rome, Italy, in 2008. In 2010, he received the M.S. degree in physics at the University of Rome "La Sapienza," Rome, in collaboration with the Agenzia nazionale per le nuove tecnologie, l'energia e lo sviluppo economico sostenibile (ENEA) of Frascati, Italy, working on the refractometry on thermonuclear plasmas.

From November 2010 to February 2011 he had an internship at the Joint European Torus (JET) where he worked on data analysis and diagnostic modelization. He is currently doing his Ph.D. degree in plasma physics started in June 2011 at the Centre de recherches en Physique des Plasmas (CRPP) of the École polytechnique fédérale de Lausanne (EPFL), in Lausanne, Switzerland.

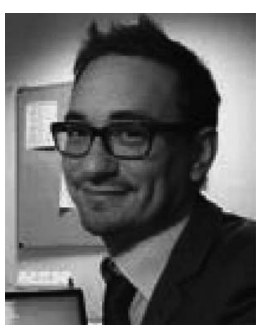

Ivan Lupelli received the M.Phil. degree in energy engineering and $\mathrm{Ph} . \mathrm{D}$., and is currently a Research Fellow at the Department of Industrial Engineering of University of Rome "Tor Vergata," Rome, Italy in the field of numerical modeling of fusion plasma physics.

Research interests include nuclear fusion safety, plasma diagnostics and statistical model for nuclear fusion data analysis.
F. P. Orsitto, photograph and biography not available at the time of publication.

O. Tudisco, photograph and biography not available at the time of publication. 